\title{
FORMATION OF ANODIC POROUS ALUMINA IN OXALIC ACID AND
} ELECTRODEPOSITION OF NI.

\author{
Khamidov Anvar \\ Doctorate of 2-course of the \\ National University of Uzbekistan \\ Nurmanov Suvankul Erhanovich \\ Doctor of technical sciences, professor, \\ Faculty of chemistry of the National University of Uzbekistan \\ Ruzimuradov Olim Norbekovich \\ Doctor of chemical sciences, professor, \\ Faculty of chemistry of the National University of Uzbekistan \\ Parmonov Askar Basimovich \\ Doctor of philosophy sciences (PhD), Faculty \\ of Chemistry of National University of Uzbekistan \\ DOI: 10.31618/nas.2413-5291.2020.2.60.303
}

\section{ABSTRACT}

Nanoporous anodic aluminum oxide (AAO) tubular membranes were fabricated from aluminum alloy tubes in sulfuric and oxalic acid electrolytes using a two-step anodization process. The membranes were investigated for characteristics such as pore size, interpore distance and thickness by varying applied voltage and electrolyte concentration. Morphology of the membranes was examined using light optical and scanning electron microscopy and characterized using Image software. Results showed that membranes having narrow pore size and uniform pore distribution with parallel channel arrays were obtained. The pore sizes were ranging from 10 to $100 \mathrm{~nm}$ and the wall thicknesses $60 \mu \mathrm{m}$.

The catalysts are obtained by impregnation of $3 \mathrm{~d}$ metals into nanosized pores of aluminum oxide. The obtained catalysts based on nickel and porous $\mathrm{Al}_{2} \mathrm{O}_{3}$ are studied by scanning microscopy (SEM-EDX). The results of SEM-EDX analysis shows that a spongy structure with filament sizes of 100 nanometers containing particles of $3 \mathrm{~d}$ metals formed on the surface of the aluminum oxide matrix.

Keywords. Nanoporous anodic aluminum oxide (AAO), carbon nanotube (CNT), tubular membrane, thickness, anodising, porous aluminium oxide film.

1. Introduction

Electrochemical oxidation, also known as anodizing, imparts an integral corrosion and abrasion resistant coating on industrially important metals. Aluminum and aluminum alloys have been treated using anodizing for nearly a century. These metals react spontaneously with ambient oxygen to form a thin oxide coating. Unlike other metal oxides, aluminum oxide does not flake; the oxide adheres to the metal preventing further oxidation. To exploit the corrosion and abrasion protection provided by aluminum oxide, forced thickening of the coating is accomplished electrochemically.

Anodization is an electrochemical oxidation process employed to increase the thickness of the native oxide layer on the surface of metals (e.g., Al, Ti, Hf, W, $\mathrm{Nb}, \mathrm{Sn}, \mathrm{Zr}$, etc.) or semiconduc-tors (e.g., Si, InP, GaAs, etc.) [1].

Nanoporous anodic aluminum oxide (AAO) has become a commonly used material with potential applications in a wide range of areas, such as catalysis, electronics, photonics, and sensing. Owing to their regular structures and narrow size distributions of pore diameters and interpore spacings, porous alumina membranes are used in the fabrication of nanometerscale composites. The nanoporous AAO sheet membrane has been investigated for a potential application in hemodialysis by measuring the hydraulic conductivity and comparing it to those of hollow fiber polymer dialysis membranes. It is known that carefully controlled anodization of aluminum in an acidic electrolyte produces a thin layer of dense aluminum oxide, followed by an ordered array of smaller-sized nanopores [2,3].

The main disadvantage of the catalysts based on nickel nanoparticles is the rapid deactivation of the catalyst due to various factors, for example, coke deposits. To prevent agglomeration and sintering, the active phase is applied to various inorganic substrates - $\mathrm{Al}_{2} \mathrm{O}_{3}, \mathrm{SiO}_{2}$, and $\mathrm{CaCO}_{3}$ [4]. The use of films, for example, $\mathrm{Al}_{2} \mathrm{O}_{3}$ as a matrix, allows combining the flexibility of the electrochemical method for obtaining catalysts and stabilizing nanoparticles in the inert matrix of porous aluminum oxide. Catalysts on substrates have several advantages, but they have low thermal conductivity. In this regard, it is relevant to create and study of nickel catalysts on metal substrates, to identify patterns of influence of synthesis conditions on the surface structure and to establish the relationship among synthesis conditions, surface structure, and such catalytic properties as an activity, stability of activity and resistance to carbonization of catalysts in the hydrogenation of olefins. Also, it is very important to have a developed surface for all metal catalysts, i.e. search the ways to increase their specific surface area.

Indeed, the establishment of their structure and nature of interaction with reagent molecules provides valuable information on the mechanisms of catalytic processes. It is the substrate that largely determines the size, shape, and the electronic state of the deposited particle [5-7].

Anodic aluminum oxide AAO, which is usually prepared by the anodic oxidation of $\mathrm{Al}$ in an acid solution [usually sulfuric $\left(\mathrm{H}_{2} \mathrm{SO}_{4}\right)$, oxalic $\left(\mathrm{H}_{2} \mathrm{C}_{2} \mathrm{O}_{4}\right)$, or 
phosphoric $\left(\mathrm{H}_{3} \mathrm{PO}_{4}\right)$ acids] [6], is one of the typical selforganized structures with a nanochannel array. Thus AAO has been commonly used to fabricate nanometersized structures via the template-mediated process because of its relatively low cost and ease of fabrication compared with conventional lithography-processed materials [8].

Recently, many researches have been focused on the nanostructured materials due to some of their significant physical properties. Although several techniques like photolithography, etching, or gas phase synthesis can produce nanowires or nanotubes, a template-assisted growing approach of nanoporous AAO is considered as one of the most prominent methods due to the advantages of a controllable diameter, high aspect ratio, and economical way in producing [9].

In this study, acid solution was used to create the aluminium oxide film with controlled morphology. However, the porous aluminium oxide film properties is strictly influenced by anodising process parameter such as anodising voltage, electrolyte temperature, acid concentration and duration of anodising process. Thus, in this study new hypothesis will be generated with exploring the relationship between electrolyte temperature and formation of aluminium oxide film on aluminium substrate in order to enlarge its applications.

\section{Experimental part}

In our experiment a high purity aluminum foil (99.999\% purity, $5 \mathrm{~mm}$ thickness) was used as a starting material. Prior to anodizing the aluminum foil was annealed at $500{ }^{\circ} \mathrm{C}$ in air for $2 \mathrm{~h}$ and degreased in acetone. Then the samples were electropolished at room temperature in 1:4 volume mixture of $\mathrm{HClO}_{4}$ and ethanol at constant current density at $25 \mathrm{~mA} / \mathrm{cm}^{2}$ for 5 $\mathrm{min}$. The hard anodization technique was applied using oxalic acid containing 0.1 M as electrolyte. During the anodization the electrolyte temperature of all samples was kept constant at $20-25^{\circ} \mathrm{C}$. This anodising process was done at $40 \mathrm{~V}$ with temperature for 120 minutes.

Anodizing of Al-plates is carried out in a twoelectrode chemical cell which is a Teflon glass with 4 sm opening in diameter in this area and mounted on a brass stand (Fig. 1).

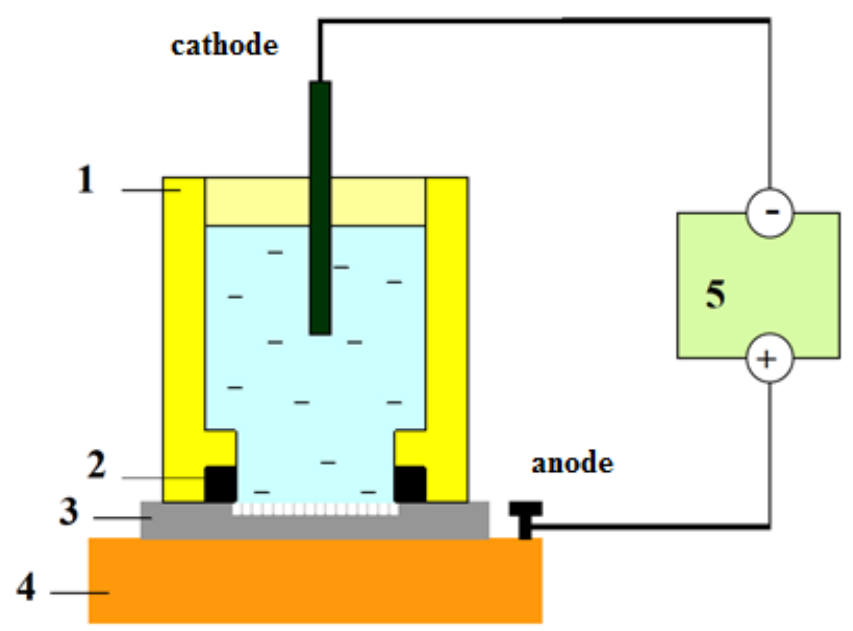

Figure 1. Experimental equipment used to produce anodized aluminum oxide.

1-Teflon glass, 2-rubber seal, 3-sample (Al-foil), 4-brass base, 5-voltage source.

The conditions of anodic oxidation depending on the selected electrolyte is given in Table 1.

Table 1.

The conditions of anodic oxidation

\begin{tabular}{|c|c|c|c|}
\hline Oxidation steps & Electrolyte & $\begin{array}{c}\text { Anodizing voltage and current, B; } \\
\text { A }\end{array}$ & $\begin{array}{c}\text { The duration of oxidation, } \\
\text { hours }\end{array}$ \\
\hline The first oxidation & $\begin{array}{c}0,1 \mathrm{M} \\
(\mathrm{COOH})_{2}\end{array}$ & $40 ; 2$ & 1 \\
\hline $\begin{array}{c}\text { The second } \\
\text { oxidation }\end{array}$ & $\begin{array}{c}0,1 \mathrm{M} \\
(\mathrm{COOH})_{2}\end{array}$ & $40 ; 2$ & 1 \\
\hline
\end{tabular}

The preanodization step for 10 min was applied to produce a thin porous oxide layer (about $500 \mathrm{~nm}$ thick) to create a protective layer against burning at high voltages [10]. As we know in each electrolyte mixture [11] the appropriate voltage for pre-anodization is related to the current and we choose this voltage such that the current density to be in the range of $1.5 \mathrm{~mA} / \mathrm{cm}^{2}$ $<\mathrm{J}<2.5 \mathrm{~mA} / \mathrm{cm}^{2}$. After preanodization (in voltage lower than $40 \mathrm{~V}$ ), the anodization voltage was increased to a final constant value by a suitable rate that is exactly related to the concentration of sulfuric acid [11]. To investigate themorphology and self ordering degree of nanopores, the SEM images were taken from the $\mathrm{Al}$ surface (imprint barrier layer) after selective etching of the porous alumina film by a mixture of $6 \mathrm{wt} . \% \mathrm{H}_{3} \mathrm{PO}_{4}$ and $1.8 \% \mathrm{H}_{2} \mathrm{SO}_{4}$ at the end of each process. The morphology and structure of porous aluminium oxide film obtained were characterised by Scanning electron microscope (SEM) model NVision 40-38-50 SEM. 


\section{Electrodeposition}

Metal/metal alloy can also be filled into the nanochannels of AAO-CNTs using a conventional electrodeposition technique. When an electric field is applied, metal ions in the electrolyte solution migrate and are deposited onto an electrode by passing a current through an electrochemical cell. Therefore, metals can be filled into the cavity of AAOCNTs by using the electrodeposition method under a direct current [15-17] or alternating current [18]. In general, a layer of $\mathrm{Au}$ is first deposited on the surface of a both-side open AAO film using ion sputtering technique. Then, metal foreigner can be deposited into the hollow core of AAOCNTs by using the pre-deposited Au layer and graphite plate as working-electrode and counterelectrode, respectively. However, it is rather difficult to handle during the electrodepositon process owing to the fragile property of AAO films.

The synthesis of nickel nanoparticles into a matrix is carried out by the method of periodic dipping with sequential drying. The aluminum oxide porous plate is alternately dipped in aqueous solutions of $\mathrm{Ni}\left(\mathrm{NO}_{3}\right)_{2}$, then it is washed in water, dried and again immersed in the solution. After a certain number of impregnation cycles, the plate is annealed in the air at $550^{\circ} \mathrm{C}$.

Basic geometric parameters of experimental samples and parameters of the surface morphology of Ni columns for samples. (Table 2.)

Table 2.

\begin{tabular}{|c|c|c|c|}
\hline Properties & Diameter pore, $\mathrm{nm}$ & Interpore diameter, $\mathrm{nm}$ & Cross-section pore, $\mu \mathrm{m}$ \\
\hline $\mathrm{AAO}$ & $90 \pm 25$ & $90 \pm 25$ & $80 \pm 20$ \\
\hline
\end{tabular}
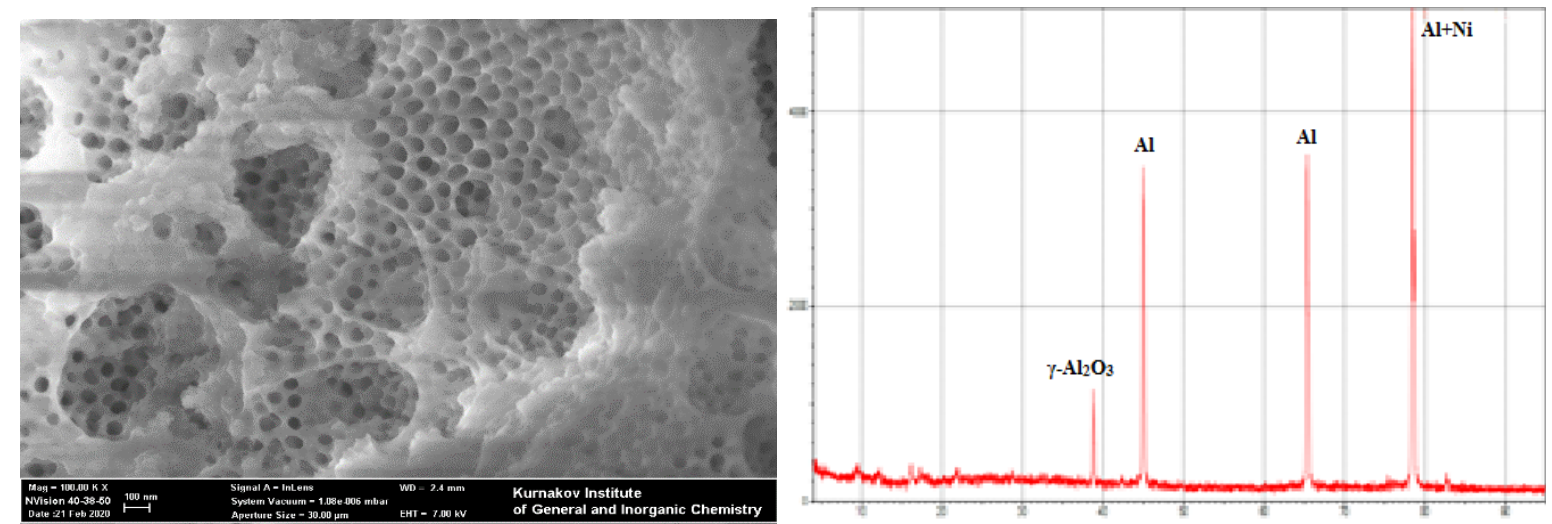

Figure 2. XRD analysis diffraction of aluminium substrate.

\section{Results and discussion}

The advantage of using porous alumina as a template is that it enables selected nano-pore fabrication using general laboratory equipment, see Figure 1. The proportionality of the cell size with respect to the electrochemical conditions allows one to macroscopically dictate the growth and final size of the pores manufactured. The equation is a simple one where an $\mathrm{Al}$ substrate is used with a particular acid to achieve nano-pores with an empirical derived set of electrochemical conditions.

$$
\mathrm{Al}+\text { acid }+ \text { voltage }=\text { nano-pores }
$$

Initially, the quality of the Al substrate, its surface structure and/or any surface pre-treatments will have a significant impact on the morphology and the resulting nano-structures formed on the substrate surface during the anodization process. To begin with, the $\mathrm{Al}$ substrate will have a pre-existing oxide layer over its surface, which is normally produced by the ambient oxygen in the atmosphere. In addition, the substrate could also have a pre-existing surface structure produced by a mechanical, thermal, chemical and electrochemical process. All of these surface treatments prior to anodization can have a significant impact on the selfordering of the pore structures that form on the surface of the substrate during the anodization process. This is because the pore nucleation mechanism is a combination of both random nucleation and nucleation produced by the effects of surface defects, such as scratches, pits, impurities and grain boundaries. It should also be pointed out that during the anodization process, surface defects are favored sites for pore nucleation. Furthermore, studies have shown that the presence of alloying elements in the Al substrate not only tend to reduce the rate of growth of the forming oxide layer, but also influence the structure of the oxide layer during the anodization process [12]. 


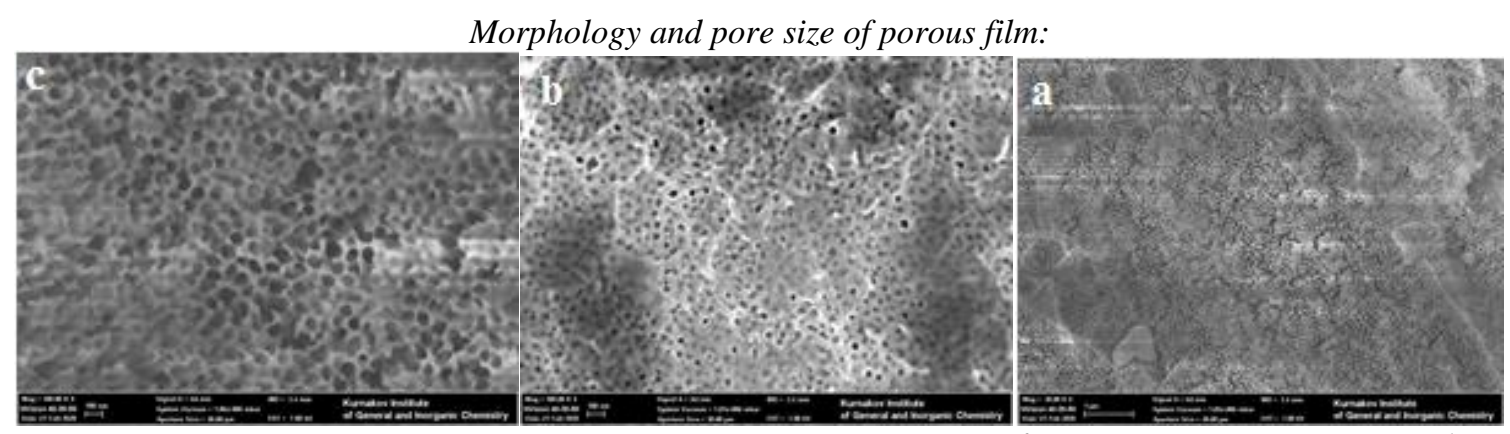

Figure 2 shows the morphology of the film anodised in temperatures, from $20^{\circ} \mathrm{C}$ to $25^{\circ} \mathrm{C}$ respectively. Figure 3: SEM images of aluminium oxide films formed in (COOH)2 at 20-25 ${ }^{\circ} \mathrm{C}$.

The average pore diameter anodised in $20-25^{\circ} \mathrm{C}$ was $100 \mathrm{~nm}$. The pores were more irregular and smaller. This trend of result is similar with Shawaqfeh [13] which has reported that the formation of oxide film in phosphate based solution was increased at temperature to $25{ }^{\circ} \mathrm{C}$, but it decreased when the temperature reached $30{ }^{\circ} \mathrm{C}$. The effect of temperature on the formation of oxide is well-defined because it affects the rate of ion transport across the barrier layer, the oxide dissolution from the pore wall as well as the oxide surface and heat transport rates within the pore and the bulk electrolyte.
An increase in temperature resulted to an increase in the growth rate of aluminium oxide film formed in phosphoric acid electrolyte. At temperature $20^{\circ} \mathrm{C}$ to $25^{\circ} \mathrm{C}$, the mass of oxide film increased. When the temperature rises up to $30^{\circ} \mathrm{C}$, the mass of oxide film decreased. The effect of temperature on the formation of aluminium oxide film is noticeable because the temperature affects the rate of ion transport across the oxide layer, the oxide dissolution from the pore wall and heat transport rates within the pore and the electrolyte $[13,14]$.

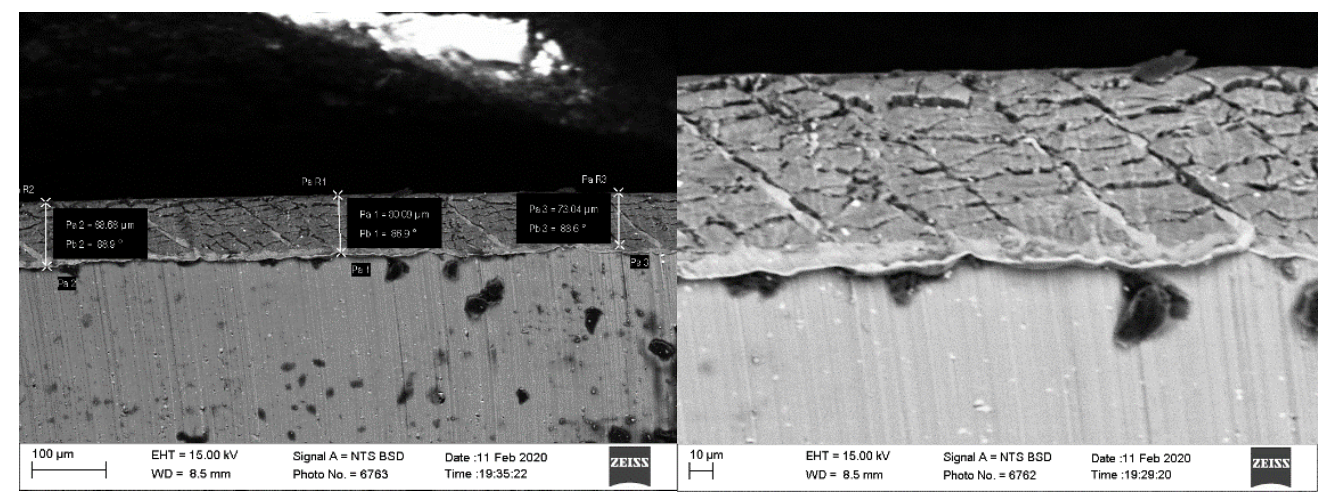

Figure 4: SEM image of cross sectional film anodised with temperature $20-25^{\circ} \mathrm{C}$.

\section{Conclusion}

In summary, the nanostructured catalysts based on $3 \mathrm{~d}$ metals (nickel) with a matrix of porous aluminum oxide was obtained. The morphological studies by SEM-EDX demonstrates a spongy structure with filament sizes of few nanometers is formed on the surface of the aluminum oxide matrix and this structure contains nanoparticles of nickel.

\section{References}

1. Woo Lee., (2010) The Anodization of Aluminum for Nanotechnology Applications., The journal of the Minerals, Metals \& Materials Society, vol. 62, no 6, pp. 57-63.

2. Mau-Phon Houng, Wei-Lun Lu, Tsung-Hsin Yang, and Kuan-Wei Lee.,(2014) Characterization of the Nanoporous Template Using Anodic Alumina Method., Journal of Nanomaterials.

3. Belwalkar A, Grasing E, Van Geertruyden W, Huang Z, Misiolek W.Z., Effect of Processing Parameters on Pore Structure and Thickness of Anodic
Aluminum Oxide (AAO) Tubular Membranes, NIH Public Access, vol. 319, no. 1-2, pp. 192-198.

4. Gene M.J, Miller A.V. (1983) Levitation-jet method of producing ultrafine metal powders. Surface. Physics, chemistry, mechanics vol. 2, pp. 150-154

5. Kondrat'eva TA, Morozov YuG, Chernov EA., Effect of conditions of manufacture on the properties of ultrafine nickel powder. Powder Metallurgy and Metal Ceramics 26 (10):793-795.

6. Gusev AI (2007) Nanomaterials, nanostructures, nanotechnologies. Fizmatlit, Moscow

7. Lee W; Nielsch K; Gösele U (2007) Selfordering behavior of nanoporous anodic aluminum oxide (AAO) in malonic acid anodization. Nanotechnology 2007, 18(47):475713. https://doi.org/10.1088/0957-4484/18/47/475713

8. Wu M.T, Leu I.C, Hon M.H., (2002) Effect of polishing pretreatment on the fabrication of ordered nanopore arrays on aluminum foils by anodization, Journal of vacuum science \& technology B, vol. 20, no.3, pp. 776-783. 
9. Chen-Kuei Chung, Ming-Wei Liao, Chun-Te Lee and Hao-Chin Chang., (2011) Anodization of nanoporous alumina on impurityinduced hemisphere curved surface of aluminum at room temperature, Nanoscale Research Letters.

10. Chu S.Z, Wada $\mathrm{K}$, Inoue $\mathrm{S}$, Isogai $\mathrm{M}$, Yasumori A., (2005) Fabrication of Ideally Ordered Nanoporous Alumina Films and Integrated Alumina Nanotubule Arrays by High-Field Anodization, Advanced materials, vol. 17, pp. 2115-2119.

11. Almasi Kashi M, Ramazani A, Mayamai Y, Noormohammadi M., (2010) Fabrication of SelfOrdered Nanoporous Alumina with 69-115 nm Interpore Distances in Sulfuric/Oxalic Acid Mixtures by Hard Anodization, Japanese Journal of Applied Physics, vol. 49, no. 1.

12. Zaraska, L, Sulka, G.D, Szeremeta J, Jaskula M., (2010) Porous anodic alumina formed by anodisation of aluminium alloy (AA1050) and high purity aluminium. Electrochimica Acta, vol. 55, no. 14, pp. 4377-4386

13. Ahmad Taleb Shawaqfeh., (1997) Fabrication and Characterization of Novel Anodic Alumina Membranes, Ph.D Thesis, Clarkson University.
14. Juyana A Wahab, Mohd Nazree Derman., (2011) Characterization of Porous Anodic Aluminium Oxide Film on Aluminium Templates Formed in Anodizing Process. Advanced Materials Research, vol. 173, pp. 55-60.

15. Wang $X \mathrm{H}$, Orikasa $\mathrm{H}$, Inokuma $\mathrm{N}$, et al., (2007) Controlled filling of Permalloy into one-endopened carbon nanotubes. Journal Material Chemistry, vol. 17, pp. 986-991.

16. Orikasa $\mathrm{H}$, Inokuma $\mathrm{N}$, Ittisanronnachai S., (2008) Template synthesis of water-dispersible and magnetically responsive carbon nano test tubes., Chem Commun, vol. 44, pp. 2215-2217.

17. Kim K H, Yamaguchi M, Orikasa H, et al. (2006) Broadband $\mathrm{GHz}$ frequency characterizations of Permalloy nanorods' array., Solid State Commun, vol. 140, pp. 491-494.

18. Czerw R, Terrones M, Charlier J C, et al., (2009) Identification of electron donor states in Ndoped carbon nanotubes., Nano Letter, vol. 9, pp. 457460. 